\title{
Detection of debonding in adhesive joints using Lamb wave propagation
}

\author{
Magdalena Rucka*, Erwin Wojtczak, and Jacek Lachowicz \\ ${ }^{1}$ Department of Mechanics of Materials and Structures, Faculty of Civil and Environmental Engineering, Gdańsk University of \\ Technology, Narutowicza 11/12, 80-233 Gdańsk, Poland
}

\begin{abstract}
Adhesively bonded joints are widely used in many branches of industry. Mechanical degradation of this type of connections does not have significant symptoms that can be noticed during visual assessment, so non-destructive testing becomes a very important issue. The paper deals with experimental investigations of adhesively bonded steel plates with different defects. Five samples (an intact one and four with damages in the form of partial debonding) were prepared. The inspection was conducted with the use of guided wave propagation method. Lamb waves were excited at one point of the sample, whereas the out-of-plane velocity signals were recorded in a number of points spread over the area of overlap. The processing of signals consisted of calculations of weighted root mean square (WRMS). The results of the analysis showed that the WRMS maps allow for identification and determination of size and shape of debonding areas.
\end{abstract}

\section{Introduction}

Metallic structures are usually assembled by bolting or welding. In recent years, there has also been a growing interest in the use of adhesive bonding in joining structural elements, especially in the aviation, machine and automotive industry [1] or even civil engineering [2]. Adhesive joints have many advantages: the stress can be more uniformly distributed and the stress concentration can be avoided, the structure weight and consequently the entire cost can be reduced, the improvement of aerodynamic features and visual appearance can be achieved [1,3]. On the other hand, adhesive joints are highly susceptible to any defects made during the preparation and joining of elements. In general, in adhesive joints three fundamental types of defects can occur: voids, inclusions and kissing defects [4]. Voids and inclusions can be efficiently detected using various non-destructive testing techniques, e.g. ultrasonic waves [5-7], thermography [4], radiography [6], digital image correlation [8-9] or electromechanical impedance spectroscopy [10]. Another group of defects are so called kissing bonds. Such defects result from the improper adhesion of the adhesive to adherend [4] and they appear when the adhesive and adherend are in intimate contact [11]. Since they are low volume defects, they are more difficult to detect by typical NDT techniques.

This paper is devoted to damage detection in a single lap adhesive joint of steel plates. Two types of debonding were investigated, namely voids and kissing defects. The inspection was based on the guided wave propagation. Lamb waves were excited at one point of the joint by a piezoactuator and measured at the selected joint area by a scanning laser Doppler vibrometry (SLDV) technique. Damage imaging was performed using the weighted root mean square (WRMS) calculations.

\section{Theoretical background}

\subsection{Lamb waves}

The essential classification of elastic waves divides them into two groups. The first one is consisted of bulk waves that propagate in unbounded media. There exist pressure waves and shear waves (horizontal and vertical). The second group of elastic waves comprises guided waves that appear in media with boundaries. For that reason, guided waves are more applicable in real engineering structures that are mostly treated as systems of elements with finite dimensions.

The guided Lamb waves are related with the wave propagation between two parallel surfaces, i.e. they are a specific kind of guided waves that propagate in solid plates. The Lamb waves appear as an effect of multiple reflections and interference of pressure and shear horizontal waves. It is well known that they have a multimodal nature, i.e. there are two types of modes: symmetric $\left(S_{0}, S_{1}, S_{2}, \ldots\right)$ and antisymmetric $\left(A_{0}, A_{1}, A_{2}\right.$, ...), as presented in Figure 1. Names of both groups are related to the shape of the plate deformation during the wave propagation. Symmetric modes are called longitudinal modes because of significant role of displacements in longitudinal direction. Antisymmetric modes are also known as flexural modes, because the displacement occurs mainly in the transverse direction.

* Corresponding author: magdalena.rucka@pg.edu.pl 
For lower frequencies only two fundamental modes (S0 and A0) can exist, whereas higher frequencies are related to the existence of larger number of Lamb modes. (a)

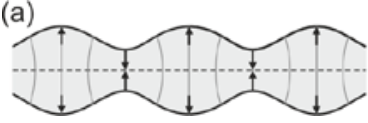

(b)

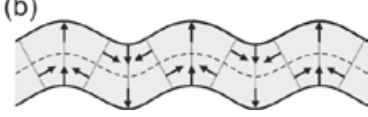

Fig. 1. Lamb wave modes: (a) symmetric; (b) antisymmetric.

What is important, the frequency value influences the propagation velocities of each existing mode. This fact proves that the Lamb waves have a dispersive nature, i.e. their characteristics are frequency dependent. The relations between frequency and other wave parameters (such as group velocity, phase velocity or wavenumber) are named dispersion relations and can be presented in a graphical form as so-called dispersion curves. There are many methods of determination of dispersion relations. A fundamental approach that can be applied for single-layer plates with a $2 d$ thickness is based on the Rayleigh-Lamb equations [12]:

$$
\begin{aligned}
& \frac{\tan \left(q_{L} d\right)}{q_{L}}+\frac{4 k^{2} p_{L} \tan \left(p_{L} d\right)}{\left(q_{L}^{2}-k^{2}\right)^{2}}=0, \\
& q_{L} \tan \left(q_{L} d\right)+\frac{\left(q_{L}^{2}-k^{2}\right)^{2} \tan \left(p_{L} d\right)}{4 k^{2} p_{L}}=0 .
\end{aligned}
$$

The method consists of searching the wavenumber values for fixed circular frequency values. Equations (1) and (2) are related to symmetric and antisymmetric modes, respectively. Parameters $q_{L}$ and $p_{L}$ depend on the wavenumber $k$, the circular frequency $\omega$ and velocities of pressure $c_{P}$ and shear waves $c_{S}$. The parameters are given by the following relations:

$$
p_{L}^{2}=\left(\frac{\omega}{c_{P}}\right)^{2}-k^{2}, \quad q_{L}^{2}=\left(\frac{\omega}{c_{S}}\right)^{2}-k^{2} .
$$

An adhesive joint can be treated as a three-layer medium. Determination of dispersion curves in multilayer plates requires more advanced calculation techniques such as matrix methods. One of many basic approaches is a transfer matrix method [13]. The multilayer medium is considered as a system of layers joined at their interfaces and immersed in vacuum. The essential of this method are the relations between amplitudes of waves propagating in one layer and stressdisplacement state at each interface adjoined to this layer. The consistency of stress and displacement values across each interface allows to determine the relation between stresses and displacement at two interfaces (top and bottom) limiting a specific layer. This relation is given utilizing a so-called layer matrix that depends on material properties and the geometry of the layer. Matrix equations for all layers can be engaged into a global relation between stresses and displacement at extreme interfaces presented by a system matrix defined as a product of layer matrices for each layer of a multilayer medium (cf. [13]). If a system is immersed in vacuum as assumed before, the extreme interfaces are stress-free. This condition allows to determine the dispersion curves for the multilayer medium. The transfer matrix method can also be applied for any single-layer medium (e.g. a homogenous plate). In this case, there is only one layer in the system, so the system matrix is identical to a layer matrix.

The dispersion curves for the analysed joint (threelayer medium) and the single-layer steel plate were calculated using authorial script prepared in MATLAB ${ }^{\circledR}$ environment with the use of the transfer matrix method. Material properties of steel were: the Young modulus $E_{s}=195.2 \mathrm{GPa}$, the Poisson's ratio $v_{s}=0.3$, the mass density $\rho_{s}=7741.7 \mathrm{~kg} / \mathrm{m}^{3}$, whereas for adhesive the values were assumed as: $E_{a}=5 \mathrm{GPa}, \quad v_{a}=0.35$, $\rho_{a}=1330 \mathrm{~kg} / \mathrm{m}^{3}$. The dispersion curves are presented in Figure 2. It is clearly visible that in the single plate (Figure $2 \mathrm{a}$ ) only two fundamental modes $\left(\mathrm{S}_{0}\right.$ and $\left.\mathrm{A}_{0}\right)$ can propagate with a considered frequency of $200 \mathrm{kHz}$. Dispersion curves for the three-layer medium (Figure 2b) reveal that for a frequency of $200 \mathrm{kHz}$ there are three modes $\left(\mathrm{S}_{0}, \mathrm{~A}_{0}\right.$ and $\left.\mathrm{A}_{1}\right)$. It can be noticed that the curve responsible for the basic antisymmetric mode $\left(\mathrm{A}_{0}\right)$ is almost identical for both systems. Also the $\mathrm{S}_{0}$ curve is similar in the vicinity of the excitation frequency. The existence of additional $A_{1}$ mode distinguishes both media. The differences between both dispersion relations are the essential for the distinction of properly bonded areas (three-layer medium and the defect surfaces (single-layer plate). For that reason, the variations in signals over good adhesion areas should vary differentially in comparison with damaged surfaces.
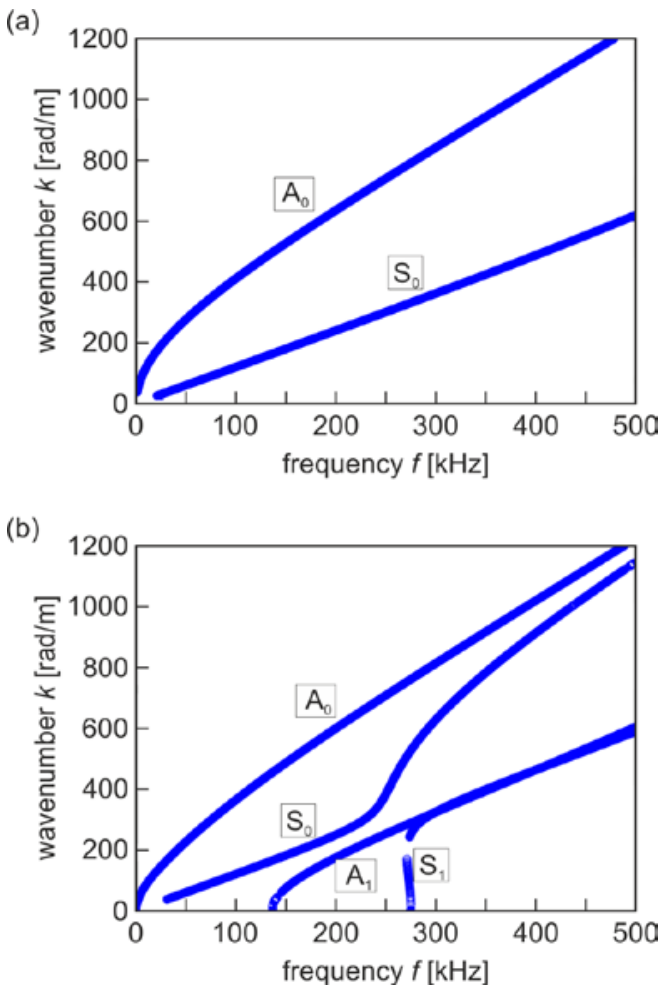

Fig. 2. Dispersion curves calculated by the transfer matrix method: (a) steel $3 \mathrm{~mm}$ plate; (b) two steel $3 \mathrm{~mm}$ plates joined with an adhesive with a thickness of $0.2 \mathrm{~mm}$. 


\subsection{Root Mean Square}

Additional processing of signals and damage imaging are required for effective determination of the actual shape and size of the internal defects. The vibration energy distribution is the basis for a simple but very efficient technique that utilizes the calculation of a root mean square (RMS). Recently, the RMS has been successfully used to detect defects in aluminium plates [14-16], delamination in a composite T-joint [17], disbonds in a stiffened panel [18], notches and corrosion areas in aluminium plates [19], disbond inserts in composite elements [20] and voids in adhesive joints [7]. The root mean square for a continuous signal $s(t)$ is calculated with respect to the formula:

$$
R M S=\sqrt{\frac{1}{t_{2}-t_{1}} \int_{t_{1}}^{t_{2}} s(t)^{2} d t},
$$

where $\left(t_{2}-t_{1}\right)$ denotes the length of the time window ( $t_{1}$ and $t_{2}$ are the beginning and the end of the time window, respectively). If analyzed parameter is represented by a discrete signal $s(t)=s_{k}$ consisted of $N$ samples recorded with a constant time interval and averaged from a time period $T=t_{2}-t_{1}$, the RMS value could be calculated from the formula:

$$
R M S=\sqrt{\frac{1}{N} \sum_{k=1}^{N} s_{k}^{2}} .
$$

An improved variant of RMS is called a weighted root mean square (WRMS):

$$
W R M S=\sqrt{\frac{1}{N} \sum_{k=1}^{N} w_{k} s_{k}^{2}} .
$$

This approach eliminates the problems with an incident signal by introducing the weighting factor $w_{k}$, i.e. the coefficient with a value depending on a number of each element of the recorded signal.

\section{Experimental investigations}

The research was conducted on a single lap adhesive joint. The adherends were steel plates, each with dimensions of $270 \mathrm{~mm} \times 120 \mathrm{~mm} \times 3 \mathrm{~mm}$. The overlap length was assumed to be the half-width of the plate $(60 \mathrm{~mm})$. The dimensions of the analyzed joint are presented in Figure 3. The process of initial preparation of plates before joining consisted of degreasing with Loctite 7063 cleaner and drying. Then the mechanical treat was applied to the surface of the overlap (abrasive paper, type P120). To avoid strength reduction, the plates were degreased again just before the application of the adhesive. Epoxy-based glue Loctite EA 9461 was used. The thickness of adhesive layer was established to be equal to about $0.2 \mathrm{~mm}$. There were three types of joints (Figure 4), an intact one (\#A) and two variants of damages in a form of voids (\#B, \#C). The defects were performed by omitting a glue layer on a part of the overlap. A PTFE tape was used to prevent the adhesive from leaking into disbonded areas.

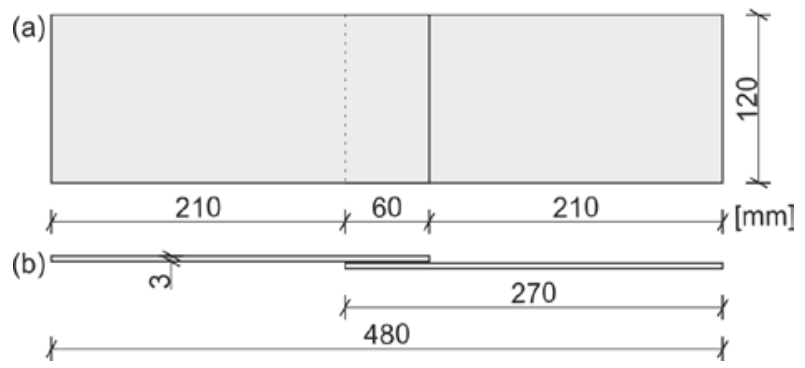

Fig. 3. The geometry of analysed adhesive joint: (a) plane view; (b) side view.

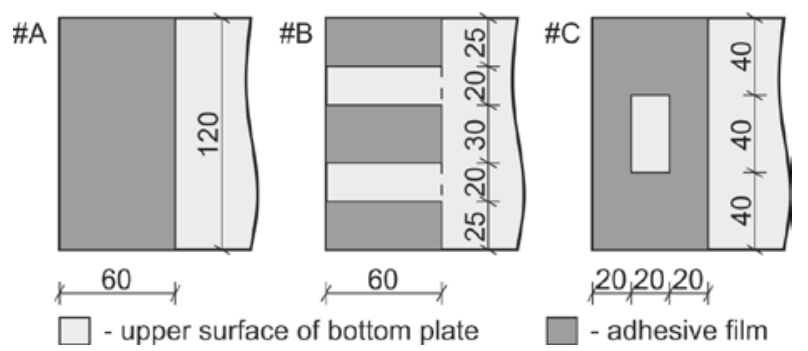

Fig. 4. The variants of analysed joints (\#A - intact joint, \#B, \#C - damaged joints).

The actual state of prepared adhesive joints was examined after experimental measurements to verify if there were any inaccuracies in the preparation process. For that reason the adherends of each specimen were separated mechanically. As for specimen \#A (Figure 5) no defects were noticed what indicated on a correct preparation. For damaged joints the designed void defects were identified. Additionally, unintended kissing bonds were visible at the surface of the overlap (where a good adhesion was designed). The existence of these damaged areas could affect the results of experimental research, so the location and shape of kissing defects was determined for each specimen type \#B and \#C (Figures 6 and 7). In this case, the ImageJ software [21] was utilized. It allowed to mark and measure the area of kissing bonds (red color lines). The surfaces of additional defects had irregular shapes and their location and size were remarkably different, even for two specimens of the same type.

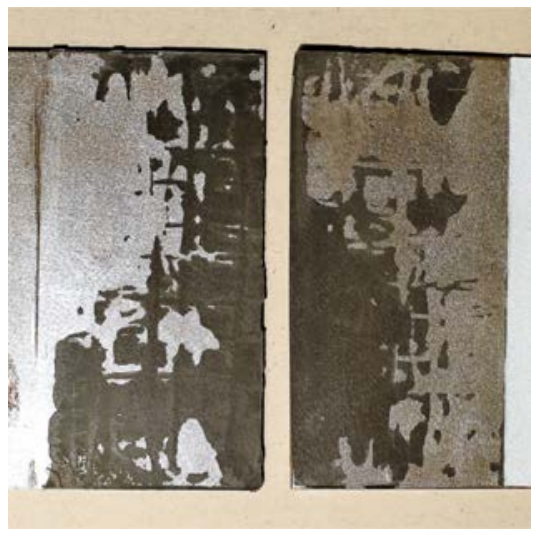

Fig. 5. Photograph of specimen \#A after separation. 
(a)

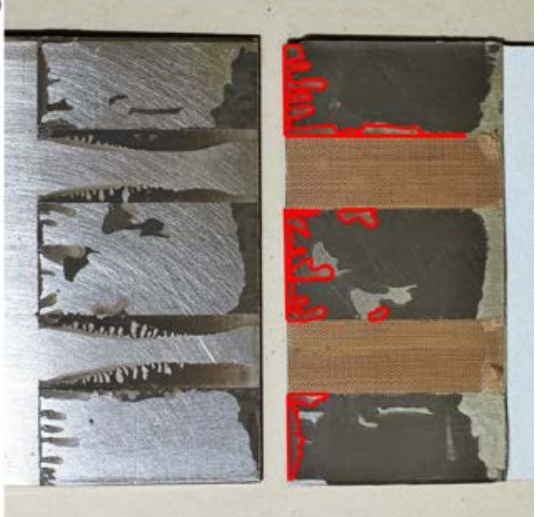

(b)

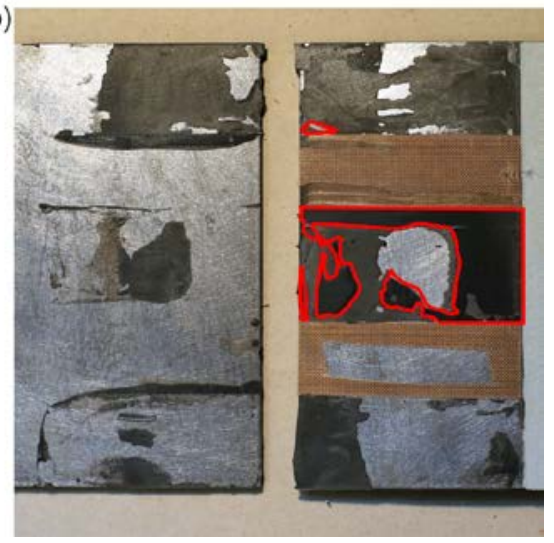

Fig. 6. Photographs of specimens of type \#B after separation (kissing bonds marked with red lines): (a) \#B.1; (b) \#B.2.

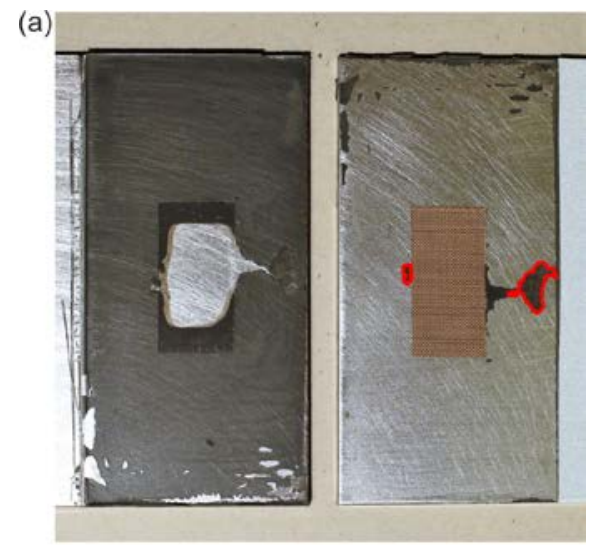

(b)

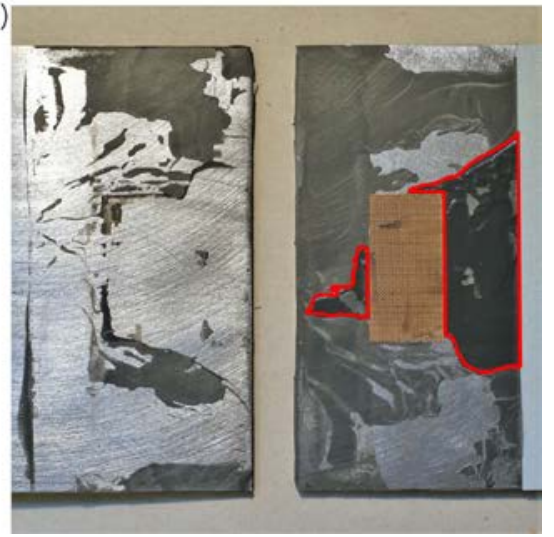

Fig. 7. Photographs of specimens of type \#C after separation (kissing bonds marked with red lines): (a) \#C.1; (b) \#C.2.
The experimental equipment shown in Figure 8a was used for the excitation of Lamb waves in one (bottom) adherend and the collection of signals at specified area of another one (top). The incident wave was generated by means of the arbitrary function generator Tektronix AFG 3022 and a high-voltage amplifier PPA 2000. The excitation signal had a form of a wave packet obtained from the five intervals of sinusoidal function with a frequency of $200 \mathrm{kHz}$ modulated by the Hanning window. The plate PZT actuator Noliac NAC2024 $(3 \mathrm{~mm} \times 3 \mathrm{~mm} \times 3 \mathrm{~mm})$ was used to excite Lamb waves. The acquisition of the guided wave propagation signals was performed by the scanning head of the laser vibrometer Polytec PSV-3D-400-M. Out-of-plane velocity components were recorded in 3721 points distributed over the top area of upper adherend in a mesh of 61 rows and 61 columns (Figure 8b).

(a)

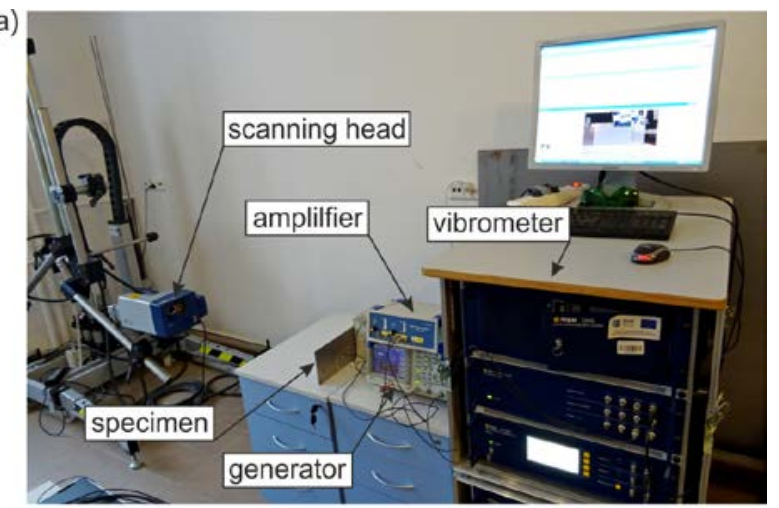

(b)

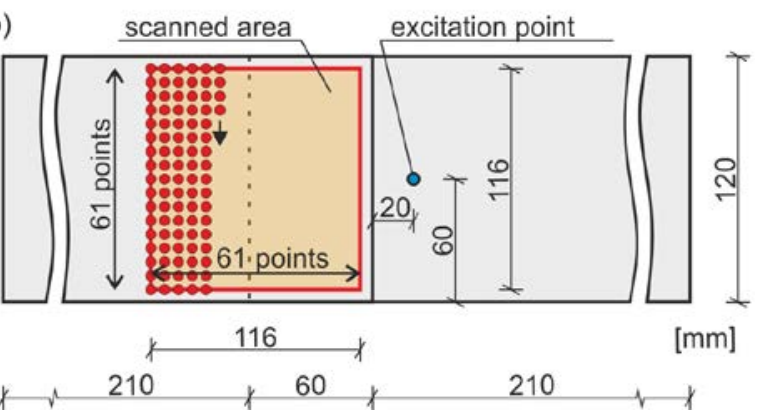

Fig. 8. Experimental setup: (a) experimental equipment; (b) measurement points.

\section{Results}

The signals collected during experimental measurements were further processed. For each one the WRMS map was calculated with respect to Eq. (6). The averaging time (time window) was assumed to be $T=3.00 \mathrm{~ms}$. A linear weighting factor was taken for calculations, i.e. $w_{k}=k$. To distinguish the areas of good adhesion and defects, the logarithmic scale was applied for all WRMS snapshots. For intact specimen \#A (Figure 9) the surface of the overlap had lower values than a single plate. The WRMS map did not allow to determine any damage areas (no disturbances at the overlap). This observation stayed in agreement with the visual investigation of the separated joint. 


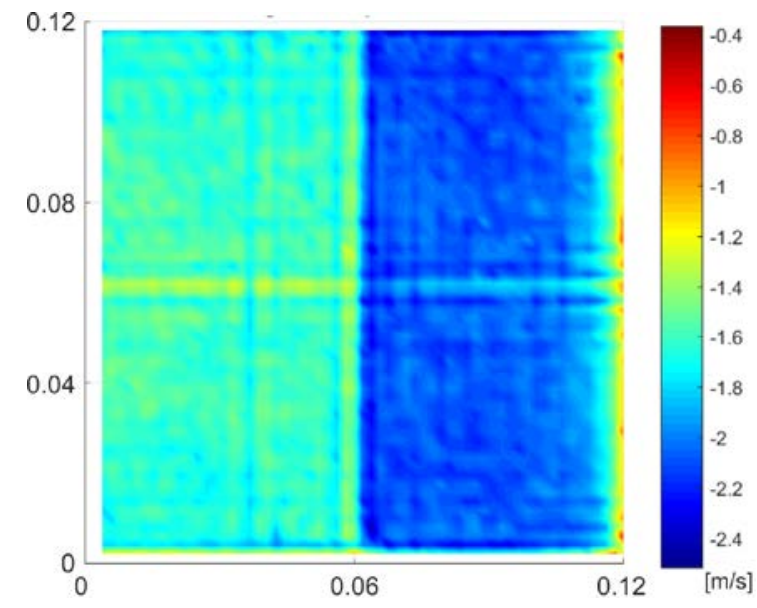

Fig. 9. WRMS map, specimen \#A.
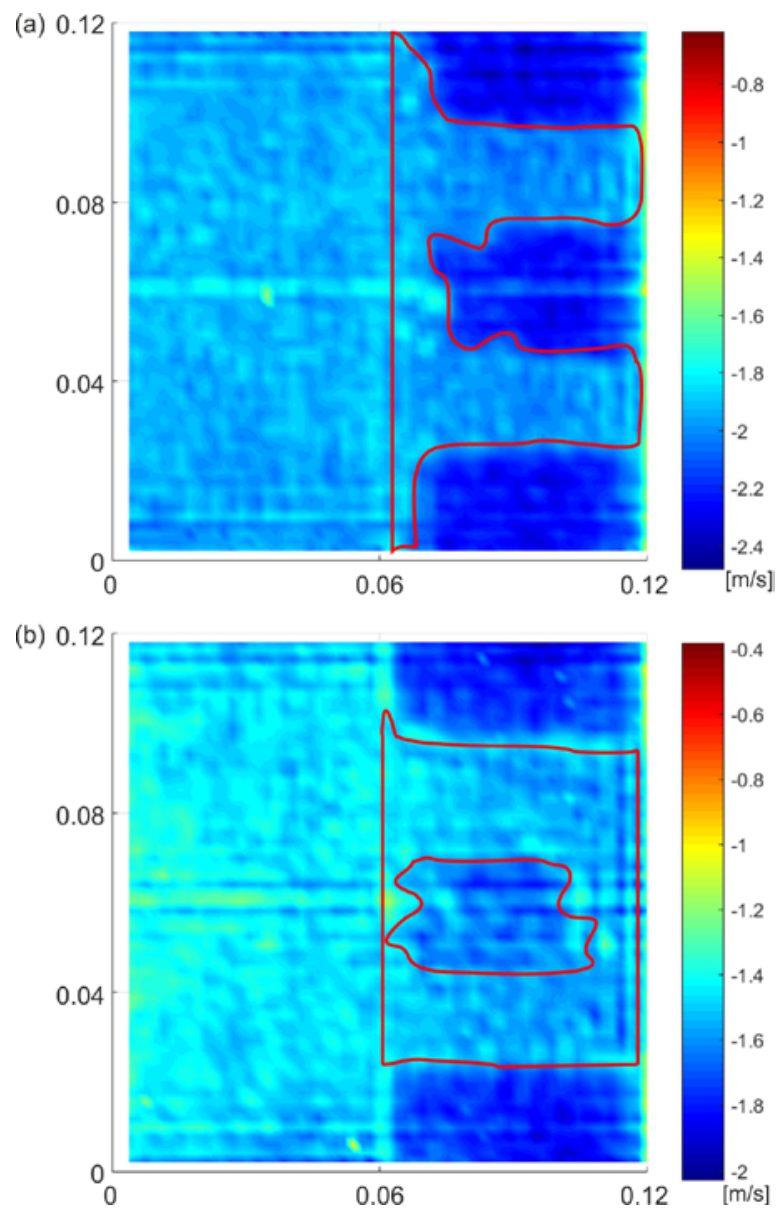

Fig. 10. WRMS maps, specimens type \#B: (a) \#B.1; (b) \#B.2.

Some interesting patterns could be observed on damaged joints (Figures 10 and 11). The defects (outlined with red lines) are clearly visible as the areas of lighter color at the overlap surfaces. The intensity of color at the defect area is close to the single plate what leads to the conclusion that there is no connection between the adherends. An important observation is that there is no possibility to distinguish voids and kissing bonds, because both defects are related to the total lack of adhesion (at a specific surface). The shape of defects identified in WRMS maps is close to that obtained from visual observations (adding voids and kissing bonds).
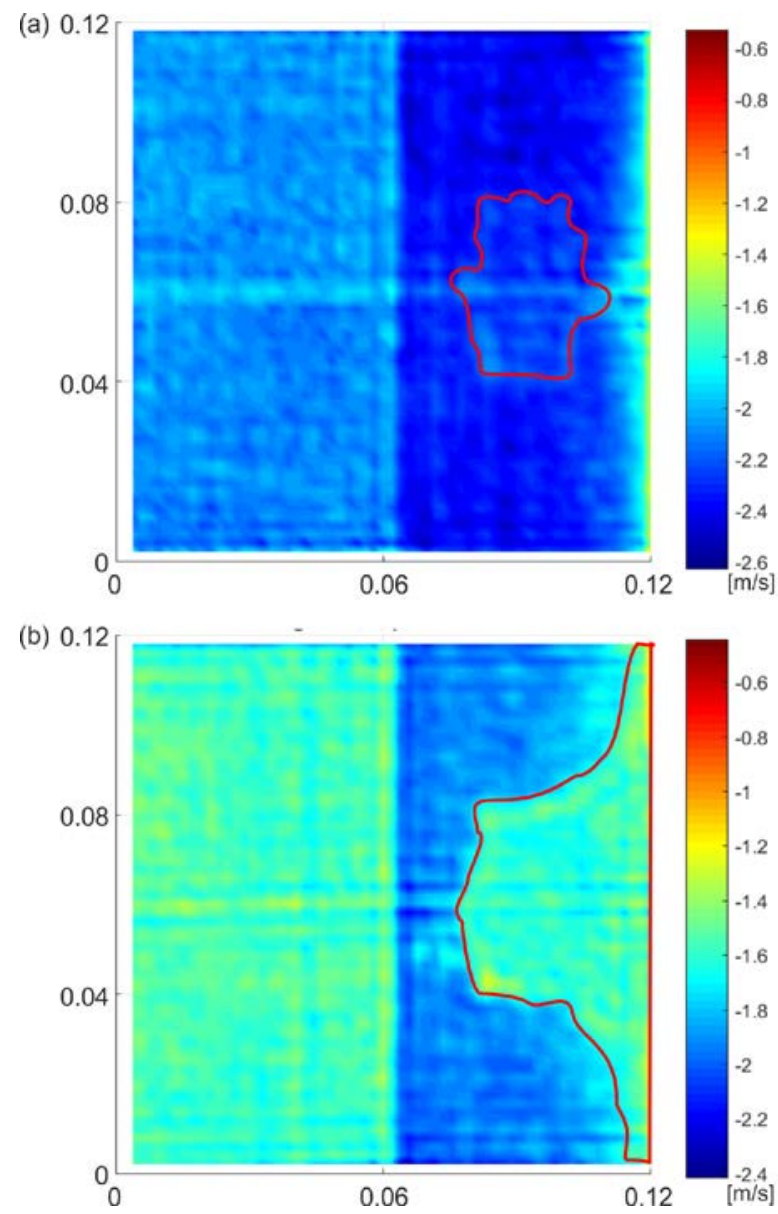

Fig. 11. WRMS maps, specimens type \#C: (a) \#C.1; (b) \#C.2.

The Image J software was used to mark and measure the actual defect surface on WRMS maps (analogically to photographs shown in Figures 6 and 7). The results of both measurements are presented in Table 1. It is visible that determined defect surface (both based on photos and WRMS maps) is greater than a designed one. The reason is the existence of kissing bonds that led to growth of damaged areas. Comparing the determined defect surface from photos and WRMS maps there are slight differences between both measurements. They may result from inaccuracies in marking the defects in ImageJ. What is more, results of measurements on specimens type \#B are similar, whereas there are significant differences between samples of type \#C (observed also visually from photos and WRMS maps).

Table 1. Designed and determined area of defects.

\begin{tabular}{|c|c|c|c|}
\hline Joint & $\begin{array}{c}\text { Designed } \\
\text { defect } \\
\text { area } \\
{\left[\mathrm{cm}^{2}\right]} \\
\end{array}$ & $\begin{array}{c}\begin{array}{c}\text { Determined } \\
\text { defect area } \\
\text { (photos) } \\
{\left[\mathrm{cm}^{2}\right]}\end{array} \\
\end{array}$ & $\begin{array}{c}\text { Determined } \\
\text { defect area } \\
\text { (WRMS maps) } \\
{\left[\mathrm{cm}^{2}\right]} \\
\end{array}$ \\
\hline \#B.1 & 24 & 28.21 & 33.41 \\
\hline \#B.2 & 24 & 34.32 & 33.01 \\
\hline \#C.1 & 8 & 8.79 & 9.90 \\
\hline \#C.2 & 8 & 20.88 & 25.04 \\
\hline
\end{tabular}




\section{Conclusions}

The paper presents the results of experimental analysis of Lamb wave propagation in adhesively bonded joints of steel plates. The research consisted of a visual assessment of separated joints and calculation of WRMS maps of recorded signals. The conclusions resulting from conducted study are presented below.

- The defects existing in adhesive joints can be detected utilizing guided Lamb waves. Additional processing of signals (WRMS calculations) are an essential for damage imaging.

- Both types of defects considered in the study (voids and kissing bonds) were successfully detected, however it was not possible to distinguish a type of the defect based on WRMS maps. The analysis of photos of separated joints was helpful in this case.

- There were significant differences between total defects detected for samples of the same type. Whereas voids were identical (their shape, size and location was designed), the kissing defects appeared unintendedly with uncontrolled geometry. This effect appeared in the visual assessment of photos, the WRMS maps and in the surface measurements made by the Image J software.

- The defect areas measured from photos and from WRMS maps were slightly different. The reason could be the differentiation between marking defects and properly bonded areas in photos (different surface characterization of adhesive after separation) and in WRMS maps (different colors).

The research work was carried out within project No. 2015/19/B/ST8/00779, financed by the National Science Centre, Poland. This support is greatly acknowledged by the authors of the study.

\section{References}

1. R.D. Adams and W.C. Wake, Elsevier Applied Science Publishers, (1986)

2. M. Piekarczyk and R. Grec, Arch. Civ. Eng., 58, 309-329 (2012)

3. F. Heidarpour, M. Farahani, and P. Ghabezi, Int. J. Adhes. Adhes., 80, 128-132 (2018)

4. R.C. Tighe, J.M. Dulieu-Barton, and S. Quinn, Int. J. Adhes. Adhes., 64, 168-178 (2016)

5. M. Korzeniowski, T. Piwowarczyk, and R.G. Maev, Arch. Civ. Mech. Eng., 14, 661-670 (2014)

6. R.L. Vijayakumar, M.R. Bhat, and C.R.L. Murthy, AIP Conf. Proc., 1430, 1276-1283 (2012)

7. M. Rucka, E. Wojtczak, and J. Lachowicz, Appl. Sci., 8, 522 (2018)

8. R.L. Vijaya Kumar, M.R. Bhat, and C.R.L. Murthy, Int. J. Adhes. Adhes., 42, 60-68 (2013)

9. R.L. Vijaya kumar, M.R. Bhat, and C.R.L. Murthy, J. Adhes. Sci. Technol., 28, 893-914 (2014)

10. W. Roth and V. Giurgiutiu, Int. J. Adhes. Adhes., 73, 109-117 (2017)
11. C. Jeenjitkaew and F.J. Guild, Int. J. Adhes. Adhes., 75, 101-107 (2017)

12. J.L. Rose, Cambridge University Press, New York (2014)

13. M.J.S. Lowe, IEEE Trans. Ultrason. Ferroelectr. Freq. Control, 42, 525-542 (1995)

14. A. Żak, M. Radzieński, M. Krawczuk, and W. Ostachowicz, Smart Mater. Struct., 21, 35024 (2012)

15. C. Lee and S. Park, Shock Vib., 725030 (2014)

16. T. Jothi Saravanan, N. Gopalakrishnan, and N. Prasad Rao, Meas. J. Int. Meas. Confed., 73, 520538 (2015)

17. G.K. Geetha, D. Roy Mahapatra, S. Gopalakrishnan, and S. Hanagud, Compos. Struct., 147, 197-210 (2016)

18. R. Marks, A. Clarke, C. Featherston, C. Paget, and R. Pullin, Appl. Sci., 6, 12 (2016)

19. C. Lee, A. Zhang, B. Yu, and S. Park, Sensors (Switzerland), 17, 1224, (2017)

20. Ł. Pieczonka, Ł. Ambroziński, W.J. Staszewski, D. Barnoncel, and P. Pérès, Opt. Lasers Eng., 99, 8087 (2017)

21. M.D. Abràmoff, P.J. Magalhães, and S.J. Ram, Biophotonics Int., 11, 36-41 (2004) 